\title{
Article
}

\section{First Steps of the Mexico Twin Registry (RGMex-MexTR) at the University of Guadalajara}

\author{
Guillermo A. Cervantes-Cardona ${ }^{1}$, Tania E. Cervantes-Nápoles ${ }^{1}$, Gabino Cervantes-Guevara² ${ }^{2}$ Olga R. Manzo-Palomera ${ }^{1}$, \\ Alejandro González-Ojeda ${ }^{3}$, Gabriela Silva-González ${ }^{1}$, Clotilde Fuentes-Orozco ${ }^{3}$, Ángel Sánchez-Michel ${ }^{1}$ and \\ Adriana Nápoles-Echauri ${ }^{1}$ \\ ${ }^{1}$ University Center of Health Sciences, University of Guadalajara, Guadalajara, Jalisco, México, ${ }^{2}$ University Center of the North, University of Guadalajara, \\ Colotlán, Jalisco, México and ${ }^{3}$ Biomedical Research Unit 02 Specialty Hospital, National Medical Center of the West, Mexican Institute of Social Security, \\ Guadalajara, Jalisco, México
}

\begin{abstract}
Despite the well-known relevance of twin studies in the medical and social sciences and the growing number of twin registries throughout the world, Latin America has not fully incorporated into the twin research community. We describe the first steps taken toward developing a twin registry in Mexico: its aim, organization, recruiting potential and main short-term objectives.
\end{abstract}

Keywords: Twins; MexTR; RGMex; registry; zygosity; Mexico

(Received 30 June 2019; accepted 7 July 2019; First Published online 16 December 2019)

The Mexico Twin Registry (MexTR) is a project whose main objective is to initiate and develop a collaborative and interdisciplinary investigation program, focused on a voluntary twin registry, in order to promote research about the interplay of genetic and environmental factors on health and complex psychological processes. Additionally, the MexTR aims to attend to the social and cultural aspects emerging around the development of twins and their families in Mexico; taking into account that the Mexican society is multicultural, housing 6 ethnic groups with 92 languages or dialectal variants, which 'gives us an approximate context of cultural diversity, its heritage, the environment, its typology and social determinants, its motivations and values' (Pérez-Pérez, 2012, p. 4).

Our main aim is to implement a strategy to set forth a collaborative infrastructure for the MexTR and the collection of data for studies on health-disease determinants in twins. The MexTR will be implemented through the University Centre for Health Sciences at the University of Guadalajara (México), and will seek collaborations with other Mexican and international research infrastructures. Health and education institutions will collaborate in establishing the registry and, when possible, an associated biobank will incorporate to the project.

Following standard recommendations (Odintsova et al., 2018) the first efforts of the MexTR have been oriented to basic research objectives and strategic planning. In this sense, important progress

Author for correspondence: Guillermo A. Cervantes-Cardona, Email: gacervantes66@ hotmail.com

Cite this article: Cervantes-Cardona GA, Cervantes-Nápoles TE, CervantesGuevara G, Manzo-Palomera OR, González-Ojeda A, Silva-González G, FuentesOrozco C, Sánchez-Michel Ángel, and Nápoles-Echauri A. (2019) First Steps of the Mexico Twin Registry (RGMex-MexTR) at the University of Guadalajara. Twin Research and Human Genetics 22: 609-610, https://doi.org/10.1017/thg.2019.95 has been made based on international collaboration and advice from the Murcia Twin Registry (Spain). The registry's recruitment scheme relies on the databases of the University of Guadalajara (State of Jalisco, Mexico), which contain records of all university students in the state. The main target population comprises all subjects, 18 years or older, who have registered in public databases in the last 20 years. As Mexican universities are in charge of organizing the educational system from secondary education onwards, this target population rises to over 1 million subjects. Identification of twins will be done by comparing two family names and the date of birth of registered individuals. In addition, collaboration agreements with the Association of Multiple Births AC and Maternity Hospital Lopez-Mateos are underway in order to facilitate access to twins from different ages and origins. Potential twins will be approached and asked to fill in a simple internet questionnaire on zygosity, self-reported BMI, self-rated health, wellbeing and happiness. The purpose of the questionnaire at this stage is not to collect data but to identify likely twins and engage them for future projects.

\section{The First Steps of the MexTR}

The development of the MexTR will follow some initial steps of an administrative and organizational nature in order to meet our scientific objectives, as well as to fulfill all the legal requirements for this kind of infrastructure in Mexico. Those first steps can be described in the following points:

(1) The generation of a relational database containing three groups of data: personal contact identification (names, surnames, address, state, municipality, town, contact telephone, email), registration and participation record (date of entry, 
participation in research or collaboration limitations) and survey data for research.

(2) The design and registration of a corporate identity in order to facilitate identification of the MexTR by the twins. This design was carried out taking into account the requirements established by the norm for the registration of trademarks and patents in our country.

(3) The official validation of a project named 'Creation of the registry of twins of Mexico', dependent of the academic body UDG-CA 832 'Psychology of Health' at the University of Guadalajara.

\section{Ethical Issues}

The MexTR will comply with all the requirements for personal data protection. All participants will be asked for their informed consent to be included in the registry. Only those who voluntarily agree to participate in the registry will be included in the database. In the case of participants under 18, these rights belong to their parents or legal guardians. All participants will have access to their data and will have the right to withdraw from the registry when they consider appropriate.

\section{Recruitment}

The recruitment procedure will consist of an active process of communication with the potential twins. This process will begin with an institutional email that will be sent to all the potential twins who meet the criteria for being included in the registry, informing them of the characteristics of the study, the scope and research objectives, and the benefits of participating in the registry. Once agreed to participate, a link to an online initial questionnaire will be sent. This link will direct first to an informed consent document. Only those who agree will have access to the questionnaire. The MexTR expects to launch its first data collection wave in the last trimester of 2019.

Additionally, the MexTR is currently preparing the first contacts with twin pairs through two alternative strategies:

(1) Twin festival: This summer the MexTR will be formally presented at a meeting of the association where several hundred twin pairs are expected. Professionals from different specialties and training will be able to contact the twins and provide information about the registry, requesting participation.

(2) Newborn registry: A collaboration agreement with the Maternity Hospital López Mateos is underway in order to facilitate contact with newborn twins' parents. Professionals from the Biomedical Research Unit 02 Specialty Hospital, linked to the MexTR, and who are specialized in attending of childbirth processes and care for premature babies will contact parents in order to start registering multiple births.

\section{Zygosity Ascertainment}

The MexTR has adapted the short zygosity questionnaire validated by the Murcia Twin Registry into Mexican Spanish. This questionnaire includes 12 items about physical similarity and identification difficulties by relatives and strangers, and has shown an adequate validity when compared to DNA markers (Ordoñana et al., 2013).

\section{Collaborations}

In short term, the MexTR will only have scientific utility for its managers. In the medium and long term, once the registry is functioning and has an established database, the resource will be available for research groups interested in using samples of these characteristics. In such cases, interested research groups will be able to submit applications to an evaluation committee that is currently being established at the University of Guadalajara. Basic requirements for collaboration will be scientific interest, authorization of the corresponding ethics or biosafety committee, and safeguarding the confidentiality and individual rights of the twins.

This initiative aims to promote scientific knowledge and collaboration as well as to extend research infrastructures in México, and being an example for other Latin American countries.

Acknowledgements. We are grateful to Prof. Juan R. Ordoñana and Dr. Juan F. Sánchez-Romera, from the Murcia Twin Registry (Spain), who have provided an invaluable help in the inception and initial development of the MexTR.

\section{References}

Odintsova, V. V., Willemsen, G. H., Dolan, C. V., Hottenga, J.J., Martin, N. G., Slagboom, P. E., ... Boomsma, D. I. (2018). Establishing a twin register: An invaluable resource for (behavior) genetic, epidemiological, biomarker, and 'omics' studies. Twin Research and Human Genetics, 21, 239-252.

Ordoñana, J. R., Rebollo, I., Carrillo, E., Colodro-Conde, L., García-Palomo, F. J., González-Javier, F., . . . Pérez Riquelme, F. (2013). The Murcia Twin Registry: A population-based registry of adult multiples in Spain. Twin Research and Human Genetics, 16, 302-306.

Pérez-Pérez, J. D. (2012). Psicología del mexicano. Tialnepantla, México: Red Tercer Milenio. 